\title{
TOPOLOGY OPTIMIZATION FOR ENERGY-EFFICIENT COMMUNICATIONS IN CONSENSUS WIRELESS NETWORKS
}

\author{
Benjamín Béjar and Martin Vetterli \\ École Polytechnique Fédérale de Lausanne (EPFL) \\ Audiovisual Communications Laboratory (LCAV) \\ \{benjamin.bejarharo, martin.vetterli\} at epfl.ch
}

\begin{abstract}
Over the past years there has been an increasing interest in developing distributed computation methods over wireless networks. A new communication paradigm has emerged where distributed algorithms such as consensus have played a key role in the development of such networks. A special case are wireless sensor networks (WSN) which have found application in a large variety of problems such as environmental monitoring, surveillance, or localization, to cite a few. One major design issue in WSNs is energy efficiency. Nodes are typically battery-powered devices and thus, it is critical to make a proper use of the scarce energy resources. This fact motivates the search for optimal conditions that favor the communication environment. It is well known that the rate at which the information is spread across the network depends on the topology of the network and that finding the optimal topology is a hard combinatorial problem. However, using convex optimization tools, we propose a method that tries to find the optimal topology in a consensus wireless network that uses broadcast messages. Our results show that exploiting the broadcast nature of the wireless channel leads to more energy efficient configurations than using dedicated unicast messages and that our algorithm performs very close to the optimal solution.
\end{abstract}

Index Terms - Topology optimization, power allocation, consensus networks

\section{INTRODUCTION}

In networked systems, a number of devices can communicate and cooperate in order to perform some global task. Such systems offer an advantage over traditional centralized ones in terms of cost, robustness and scalability, making them particularly suitable for largescale data analysis and monitoring. A clear example of a networked system that has become very popular over the past years is a Wireless Sensor Network (WSN). WSNs are composed by a large number of low-cost devices (nodes) equipped with a variety of sensors to measure quantities such as temperature, humidity, motion, etc. Such nodes have limited computational and power resources and their purpose is to gather and retrieve information from the environment [1]. A new communication paradigm has emerged where smart agents can take autonomous decisions and interact with each other without the supervision of a centralized entity. The proliferation of networked systems in general, and of WSNs in particular, have motivated the development of new methods and algorithms for distributed processing. As an example, average consensus algorithms [2-6] have gained a lot of popularity in recent years and have been widely used for the integration of the acquired information across the network. Under mild connectivity conditions, average consensus algorithms appear as simple and effective mechanisms for the computation of global averages from local estimates. Furthermore, they can also be used as a basic tool for solving more general problems in a distributed fashion [7-9].

In the context of WSNs, energy efficiency is a major design issue that should be looked at carefully. It is desirable for such networks to be autonomous and capable of working for long periods of time without battery replacement. The amount of energy spent by the network is directly related to the configuration of the nodes themselves and how they communicate. In the case of a consensus network with bidirectional links (undirected graph), the rate at which information spreads across the network is related to the second smallest eigenvalue of the Laplacian matrix, also known as the algebraic connectivity of the graph. Intuitively, the algebraic connectivity of the graph gives us a measure of how well the network is connected (ie., the smaller its value the lower the connectivity of the network). For instance, it can be shown that a graph is connected if and only if its algebraic connectivity is positive [10]. As a consequence, there have been some efforts dedicated to find the optimal topology/way of mixing information over consensus networks in order to maximize the rate of convergence of consensus algorithms [11-14]. A recent approach looks at the topology optimization problem from an energy-efficient perspective [15]. The authors propose a connectivity model based on the received power at the nodes and try to find the optimal power allocation (and topology) that yields the least energy consumption in a consensus network. However, the authors in [15] only consider the case of unicast messages and do not take advantage of the broadcast nature of the wireless channel. Exploiting broadcast communications helps to preserve energy resources, hence enlarging the lifespan of the network.

In this paper, we consider the problem of topology optimization for energy-efficient consensus-type communications in WSNs using broadcast messages. We formulate the problem as a binary optimization problem on the edge variables and transmission powers. In order to solve the problem we use a relaxation of the binary variables and allow them to take values within the unit interval. We show that the objective function in the relaxed problem is quasi-convex and can be efficiently solved using a bisection search over a family of semidefinite feasibility problems. Our formulation is particularly appealing and our results easily reproducible using any general-purpose optimization package such as CVX [16]. We will show in the experimental part that our method is more energy-efficient than using unicast dedicated messages as in [15]. 


\section{PROBLEM FORMULATION}

Consider a networked system such a wireless sensor network composed of set of $n$ elements, and where each node uses broadcast-type messages to communicate with its adjacent (1-hop) neighbors. Let $p_{i}, i=1, \ldots, n$ be the transmission power used by the $i$ th node. Assume a propagation model where the received signal gets attenuated with the travelled distance so that the received power at node $j$ when node $i$ is transmitting is given by

$$
p_{i j}=\frac{p_{i}}{1+\left(\frac{r_{i j}}{r_{0}}\right)^{\alpha_{i j}}},
$$

where $r_{i j}=r_{j i}$ is the distance between nodes $i$ and $j, r_{0}$ is a reference distance where full power is received, and $\alpha_{i j}=\alpha_{j i}$ models the attenuation characteristics of the channel.

In order to allow for a reliable signal decoding, a proper threshold on a certain Quality of Service measure such as the Bit Error Rate (BER) must be imposed. A requirement in terms of BER can be equivalently expressed as a requirement in terms of the received signal power. Therefore, connectivity among the nodes is established based on whether a threshold in the received power over the link between them is met. This means that the received power in both directions $\left(p_{i j}\right.$ and $\left.p_{j i}\right)$ should be above the minimum required threshold.

More formally, let $E=\{1, \ldots, L\}$ be the set of all possible edges in the network (i.e., $L=n(n-1) / 2$ ), and let $x_{l} \in\{0,1\}$, $l \in E$, be a binary variable that takes the value 1 if the $l$ th edge (link) is active and 0 otherwise. Then, based on our propagation and connectivity models, it follows that

$$
x_{l_{i j}}=\left\{\begin{array}{ll}
1 & p_{i j} \geq p_{\text {th }}, p_{j i} \geq p_{\text {th }} \\
0 & \text { otherwise }
\end{array},\right.
$$

where $l_{i j} \in E$ corresponds to the edge that links nodes $i$ and $j$, and where $p_{\text {th }}$ represents a threshold on the received power that allows for reliable signal decoding. It is clear from (1) and (2) that fixing the transmission powers $p_{i}$ would determine the underlying graph topology, and that given a fixed topology, the minimum required transmission powers can also be determined. Also note that not all the combinations of transmission powers $p_{i}$ are allowed since by (2) we are requiring the graph to be undirected, therefore the transmission powers must be related to each other.

We are interested in minimizing the energy consumption over a consensus network that uses broadcast-type messages for communications. In order to analyze the properties of the network let us introduce some notation that will facilitate the formulation of the problem. Let $\mathbf{a}_{l} \in\{-1,0,1\}^{n}, l \in E$, be a vector such that $\mathbf{a}_{l}(i)=1$ and $\mathbf{a}_{l}(j)=-1$ if nodes $i$ and $j$ are adjacent through link $l$, and $\mathbf{a}_{l}(k)=0$ for $k \neq i, j$. The Laplacian matrix of the graph can then be expressed as a function of the edges variables $x_{l}$ as

$$
\mathbf{L}(\mathbf{x})=\sum_{l=1}^{L} x_{l} \mathbf{a}_{l} \mathbf{a}_{l}^{\top},
$$

where $\mathbf{x}=\left[x_{1}, \ldots, x_{L}\right]^{\top}$ is a vector containing all edges in the network.

Recall that the rate of convergence for gossip algorithms is related to the second smallest eigenvalue $\lambda_{2}(\mathbf{L})$ (algebraic connectivity) of the Laplacian matrix [11]. Similarly to [15], a measure of the required energy to achieve consensus over a graph using broadcasttype messages is then proportional to

$$
e \propto \frac{\mathbf{1}^{\top} \mathbf{p}}{\lambda_{2}(\mathbf{L}(\mathbf{x}))}
$$

where $\mathbf{p}=\left[p_{1}, \ldots, p_{n}\right]^{\top}$ is a vector of all transmission powers and $\mathbf{1}$ is a vector of all ones.

Taking into account (1) and (2), the relationship between the transmission powers $p_{i}$ and the corresponding variables $x_{l_{i j}}$ is given by

$$
x_{l_{i j}}=\left\{\begin{array}{ll}
1 & p_{i}, p_{j} \geq \beta_{i j} \\
0 & \text { otherwise }
\end{array},\right.
$$

where $\beta_{i j}$ is given by

$$
\beta_{i j}=\beta_{j i}=p_{\text {th }}\left[1+\left(\frac{r_{i j}}{r_{0}}\right)^{\alpha_{i j}}\right] .
$$

Note that the value of $\beta_{i j}$ represents the threshold on the transmission powers $p_{i}$ and $p_{j}$ for the $i j$ th link to be active.

Our goal is to find the topology (and transmission powers) that minimize the energy consumption in (4). Based on (5) we can now formulate the energy minimization problem as

$$
\begin{array}{cl}
\underset{\mathbf{p}, \mathbf{x}}{\operatorname{minimize}} & \frac{\mathbf{1}^{\top} \mathbf{p}}{\lambda_{2}(\mathbf{L}(\mathbf{x}))} \\
\text { subject to } & x_{l} \in\{0,1\}, l=1, \ldots, L \\
& p_{i} \geq x_{l_{i j}} \beta_{i j}, i, j=1, \ldots, n
\end{array}
$$

where the last set of constraints captures the relationship between the edge and transmission power variables.

\section{RELAXATION AND ALGORITHMS}

Problem (7) is hard to solve due to the binary constraint on the edge variable $\mathbf{x}$. Instead of dealing directly with problem (7) we will resort to a relaxed version of the problem by allowing the variable $\mathbf{x}$ to take values on the unit interval (i.e., $0 \leq x_{l} \leq 1$ ). Also note that we can get rid of variable $\mathbf{p}$ by expressing it in terms of the variable $\mathrm{x}$ using the relation

$$
p_{i}=\max _{j} \beta_{i j} x_{l_{i j}}
$$

It is easy to show that, at the optimum of problem (7), the transmission power at the nodes must satisfy equation (8).

With these considerations in mind, we can express the relaxed version of (7) as

$$
\begin{array}{ll}
\underset{\mathbf{x}}{\operatorname{minimize}} & \frac{\sum_{i=1}^{n} \max _{j} \beta_{i j} x_{l_{i j}}}{\lambda_{2}(\mathbf{L}(\mathbf{x}))} \\
\text { subject to } & 0 \leq x_{l} \leq 1, l=1, \ldots, L
\end{array}
$$

An important property of problem (9) is that it belongs to the class of quasi-convex optimization problems and thus, can be solved efficiently using numerical methods. In order to see this, consider the objective function in (9), and let us denote it by $f(\mathbf{x})$. By definition, a function $g(\mathbf{x})$ is quasi-convex if the set $\mathcal{X}=\{\mathbf{x} \mid g(\mathbf{x}) \leq \delta\}$ is convex for every $\delta \in \operatorname{range}(g(\mathbf{x}))$. Note that $f(\mathbf{x})=\frac{\sum_{i=1}^{n} \max _{j} \beta_{i j} x_{l_{i j}}}{\lambda_{2}(\mathbf{L}(\mathbf{x}))}$ only takes positive values. Therefore, we have that

$$
f(\mathbf{x}) \leq \delta \Longleftrightarrow \sum_{i=1}^{n} \max _{j} \beta_{i j} x_{l_{i j}}-\delta \lambda_{2}(\mathbf{L}(\mathbf{x})) \leq 0,
$$

where $\delta \in \mathbb{R}_{+}$is a positive number. In order to show quasiconvexity of $f(\mathbf{x})$ we need to show that the set of points defined by (10) is indeed convex. To see this, note that the left-hand-side of the second inequality in (10) consists of the addition of two terms. The first term $\sum_{i=1}^{n} \max _{j} \beta_{i j} x_{l_{i j}}$ is a convex function in $\mathbf{x}$ since it is 
the addition of convex functions (note that $\max _{j} \beta_{i j} x_{l_{i j}}$ is a convex function in $\mathbf{x})$. The second term, $-\delta \lambda_{2}(\mathbf{L}(\mathbf{x}))$ is also convex in $\mathbf{x}$ since $\lambda_{2}(\mathbf{L}(x))$ is concave in $\mathbf{x}$ and $\delta$ is positive. Concavity of $\lambda_{2}(\mathbf{L}(\mathbf{x}))$ can be easily shown since it is the pointwise infimum of a family of convex (actually, linear) functions of $\mathbf{x}$ (see, [17]), that is

$$
\lambda_{2}(\mathbf{L}(\mathbf{x}))=\inf _{\mathbf{u} \in \mathcal{U}} \mathbf{u}^{\top}\left(\sum_{l=1}^{L} x_{l} \mathbf{a}_{l} \mathbf{a}_{l}^{\top}\right) \mathbf{u},
$$

where $\mathcal{U}=\left\{\mathbf{u} \mid \mathbf{u}^{\top} \mathbf{1}=0,\|\mathbf{u}\|_{2}=1\right\}$.

Now that we have shown the quasi-convexity of $f(\mathbf{x})$, a simple method for solving problem (9) is to use a bisection search over $\delta$. This can be easily done by solving a family of feasibility problems of the form

$$
\begin{array}{ll}
\text { find } & \mathbf{x} \\
\text { subject to } & \sum_{i=1}^{n} \max _{j} \beta_{i j} x_{l_{i j}}-\delta \lambda_{2}(\mathbf{L}(\mathbf{x})) \leq 0, \\
& 0 \leq x_{l} \leq 1, l=1, \ldots, L
\end{array}
$$

Note that feasibility problem (12) is convex in the optimization variable $\mathbf{x}$. For its solution we will use a semidefinite program (SDP) formulation of the problem that can be efficiently solved with general-purpose optimization packages such as CVX [16]. It is easy to realize that problem (12) can be equivalently expressed as the following SDP feasibility problem

$$
\begin{array}{ll}
\text { find } & \{\mathbf{x}, \mathbf{p}, s\} \\
\text { subject to } & \sum_{i=1}^{n} p_{i}-\delta s \leq 0, \\
& \sum_{l=1}^{L} x_{l} \mathbf{a}_{l} \mathbf{a}_{l}^{\top}-\frac{\mathbf{1 1}^{\top}}{n} \succeq s \mathbf{I}, \\
& p_{i} \geq \beta_{i j} x_{l_{i j}}, i, j=1, \ldots, n, \\
& 0 \leq x_{l} \leq 1, l=1, \ldots, L,
\end{array}
$$

where an inequality of the form $\mathbf{A} \succeq \mathbf{B}$ means that matrix $\mathbf{A}-\mathbf{B} \succeq \mathbf{0}$ is positive semi-definite. For completeness, the bisection procedure to find the optimal solution of problem (9) is outlined in Algorithm 1, where $\varepsilon$ is a small number to decide upon convergence. Note that the $\varepsilon$-optimal value of problem (9) corresponds to the output $f^{\star}$ coming from algorithm (1).

We have now a method that allows us to find a lower bound on the optimal value of the original problem in (7). Using the solution to the relaxed problem (9) provided by the bisection search in Algorithm 1, we can find a topology by thresholding the value of $\mathbf{x}^{\star}$. However, this does not guarantee that the obtained topology is a valid one. An alternative method consist of sorting the values in $\mathrm{x}^{\star}$ in descending order and forming different topologies by adding

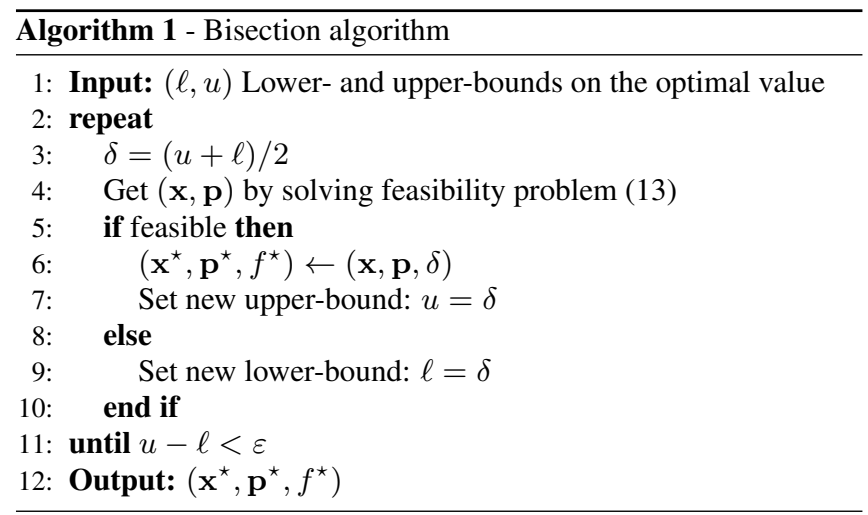

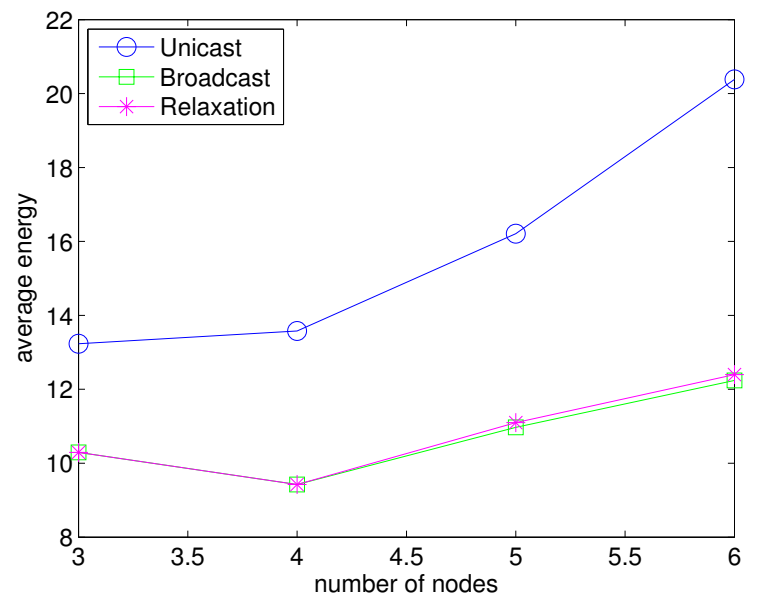

Fig. 1: Average energy consumption using unicast versus broadcast messages for different sizes of the network and for a path-loss exponent of $\alpha=3.5$.

one edge at a time. For each topology, we can easily check whether it constitutes a valid broadcast topology or not. If the topology is valid, we can then compute the objective function and keep the topology with the smallest objective. Note that such an approach requires the evaluation of at most $n(n-1) / 2$ topologies and that there will always be at least one valid topology (e.g., a fully connected network). In practice, however we have observed that the found topology using this procedure coincides most of the time with the optimal one.

\section{SIMULATIONS}

In this section we provide some numerical examples to illustrate the validity of the proposed method for optimizing the topology of a wireless consensus network using broadcast messages. For the propagation model, we have used a threshold in the minimum received power of $p_{\text {th }}=0.01$, and a reference distance of $d_{0}=0.1$. The path-loss exponent has been assumed to be the same for all links in the network (i.e., $\alpha_{i j}=\alpha$, for all $i, j$ ). All networks have been randomly generated to uniformly lie within the unit square (nodes' coordinates are drawn from a uniform distribution over the unit square). In order to illustrate the advantage of broadcast-type of communications as compared to unicast, we have run a simulation where we varied the number of nodes from $n=3$ up to $n=6$. For these numbers, it is still possible to evaluate all possible topologies and establish a back to back comparison of our method and the optimal solution. For higher number of nodes, however, an exhaustive search becomes impractical since the number of possible topologies grows as fast as $2^{\frac{n(n-1)}{2}}$. In Figure 1 we have displayed the average energy consumption over 100 random network realizations as a function of the number of nodes, and for a path-loss exponent $\alpha=3.5$. The optimal topologies for the unicast and broadcast cases have been computed (labelled as Unicast and Broadcast, respectively) and displayed in the figure. Also, the energy consumption resulting from the topology found using our approach (labelled as Relaxation) has been also included in the figure. As it can be appreciated, there is a clear advantage of broadcast communications versus unicast, an effect that becomes even more pronounced as the number of nodes increases. It is important to mention that in the unicast case we have 


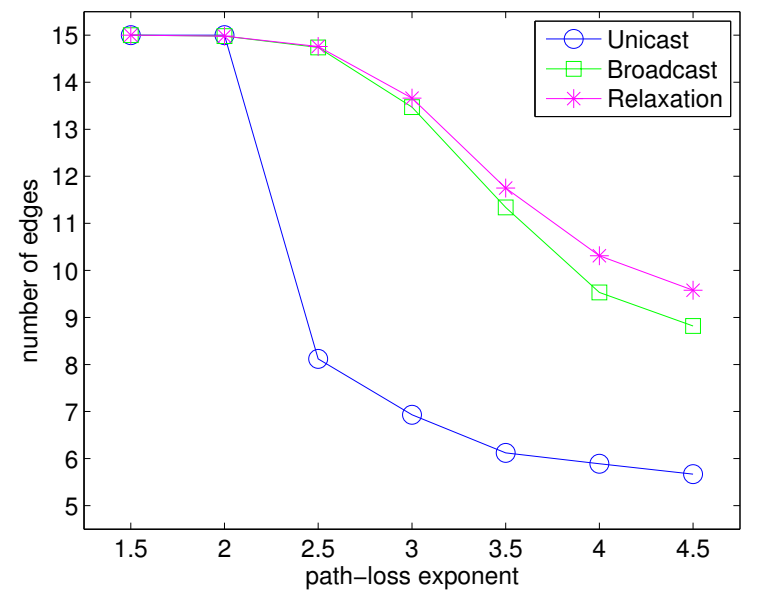

Fig. 2: Average number of edges as a function of the path-loss exponent for a network of $n=6$ nodes.

to count twice the power put on every link since the communication is bidirectional. Also note that the approach in [15] is lower bounded by the Unicast curve. When comparing the optimal solution to the broadcast scenario and the proposed method we can see that our technique comes very close to the optimal solution and, consequently, outperforms the unicast scenario and the approach in [15].

Another interesting figure to look at is the number of edges of the network. For a given network, the amount of active links should be larger when the propagation channel conditions are favorable. To formalize this intuition we have performed a simulation where we have averaged the number of active links in a network of $n=6$ nodes as a function of the path-loss exponent. The results of the experiment are depicted in Figure 2. As expected, when the channel conditions are very favorable, the best strategy is to use all links (i.e., fully connected network, 15 edges in our example), whereas the optimal topology approaches a tree (e.g., 5 edges in our example) as communication becomes more expensive. Also in Figure 2 we observe a very good agreement between the optimal topology and the one found by our method.

As an illustrative example we have also depicted in Figures 3 and 4 a fully connected network of $n=20$ nodes and the resulting topology after optimizing with our method. As it can be appreciated, there is a significant reduction in the number of links that should be used in order to minimize the energy consumption.

\section{CONCLUSIONS}

We have presented an approach for reducing the amount of energy consumed in a consensus network by finding the optimal topology (and power allocation). Our method takes advantage of broadcasttype of messages in order to reduce the energy consumption as compared to the unicast case of [15]. Numerical experiments show that the developed procedure performs close to the optimal case in the tested scenarios. Another advantage of our formulation is that it can be easily implemented with any general-purpose optimization software, making our results easily reproducible.

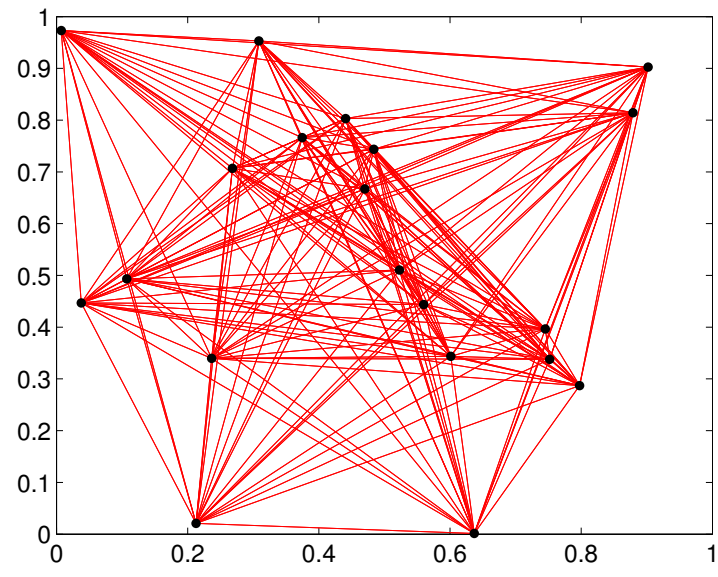

Fig. 3: A fully connected network of $n=20$ nodes.

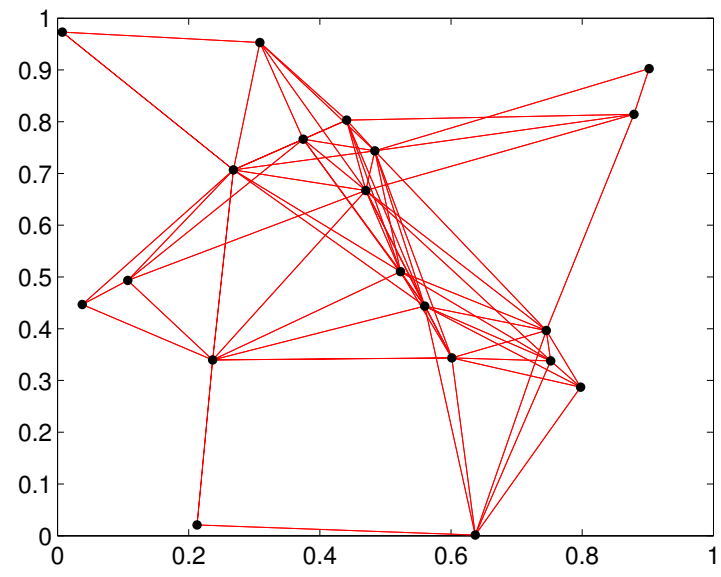

Fig. 4: Best topology obtained through our method.

\section{ACKNOWLEDGEMENTS}

This work has been conducted as part of the project HANDiCAMS www . handicams - fet . eu. The project HANDiCAMS acknowledges the financial support of the Future and Emerging Technologies (FET) programme within the Seventh Framework Programme for Research of the European Commission, under FET-Open grant number: 323944.

\section{REFERENCES}

[1] I. Akyildiz, W. Su, Y. Sankarasubramaniam, and E. Cayirci, "Wireless sensor networks: a survey," Computer Networks, vol. 38, no. 4, pp. $393-422,2002$.

[2] M. H. DeGroot, "Reaching a Consensus," Journal of the American Statistical Association, vol. 69, no. 345, pp. 118 - 121, March 1974.

[3] V. Borkar and P. Varaiya, "Asymptotic Agreement in Dis- 
tributed Estimation,", IEEE Transactions on Automatic Control, vol. 27, no. 3, pp. 650 - 655, June 1982.

[4] J. N. Tsitsiklis and D. P. Bertsekas, "Distributed Asynchronous Deterministic and Stochastic Gradient Optimization Algorithm,", IEEE Transactions on Automatic Control, vol. 31, no. 9, pp. $803-812$, September 1986.

[5] R. Olfati-Saber and R. Murray, "Consensus Problems in Networks of Agents with Switching Topology and Time-Delays," IEEE Trans. Autom. Control, vol. 49, no. 9, pp. 1520-1533, September 2004.

[6] F. Garin and L. Schenato, Networked Control Systems, ser. Lecture Notes in Control and Information Sciences. Springer, 2011, vol. 406, ch. A survey on distributed estimation and control applications using linear consensus algorithms, pp. 75107.

[7] M. G. Rabbat, R. D. Nowak, and J. A. Bucklew, "Generalized consensus computation in networked systems with erasure links," in Proc. IEEE 6th Workshop Signal Processing Advances in Wireless Communications, 2005, pp. 1088-1092.

[8] J. Li, E. Elhamifar, I.-J. Wang, and R. Vidal, "Consensus with robustness to outliers via distributed optimization," in Proc. 49th IEEE Conf. Decision and Control (CDC), 2010, pp. 21112117.

[9] S. Boyd, N. Parikh, E. Chu, B. Peleato, and J. Eckstein, Distributed Optimization and Statistical Learning via the Alternating Direction Method of Multipliers. Foundations and Trends in Machine Learning, 2011, vol. 3, no. 1.

[10] B. Mohar, "The Laplacian spectrum of graphs," in Graph Theory, Combinatorics, and Applications. Wiley, 1991, pp. 871898.

[11] L. Xiao and S. Boyd, "Fast linear iterations for distributed averaging," Systems and Control Letters, vol. 53, pp. 65-78, 2004.

[12] S. Boyd, "Convex optimization of graph laplacian eigenvalues," in in International Congress of Mathematicians, no. 3, 2006, pp. 1311-1319.

[13] S. Boyd, A. Ghosh, B. Prabhakar, and D. Shah, "Randomized gossip algorithms," IEEE/ACM Trans. Netw., vol. 14, no. SI, pp. 2508-2530, Jun. 2006. [Online]. Available: http://dx.doi.org/10.1109/TIT.2006.874516

[14] A. Ghosh and S. Boyd, "Growing well-connected graphs," in 45th Conference on Decision and Control, IEEE, ser. CDC'06, San Diego, CA, USA, Dec. 2006, pp. 6605 - 6601.

[15] S. Sardellitti, S. Barbarossa, and S. Ananthram, "Optimal topology control and power allocation for minimum energy consumption in consensus networks," Transactions on Signal Processing, IEEE, vol. 60, no. 1, pp. 383 - 399, jan. 2012.

[16] M. Grant and S. Boyd, "CVX: Matlab software for disciplined convex programming, version 2.0 beta," http://cvxr.com/cvx, Sep. 2013.

[17] S. Boyd and L. Vandenberghe, Convex Optimization. New York, NY, USA: Cambridge University Press, 2004. 